\title{
Optimisation of radiation dose and image quality in 3D multi-detector CT angiography in abdominal aortic aneurysm: an in vitro aorta phantom study
}

\author{
Kirstie McCann, Victor Lim, Guy Tomlinson, Jason Way, Ahmed Alshamakhi, Jeanette Ong, Zhonghua Sun
}

\author{
Discipline of Medical Imaging, Department of Imaging and Applied Physics, \\ Curtin University of Technology, Perth, Western Australia 6845, Australia \\ Correspondence z.sun@curtin.edu.au
}

\begin{abstract}
The aim of the study was to investigate the relationship of radiation dose and image quality in 3D multi-detector computed tomography (CT) angiography of abdominal aortic aneurysm based on an aorta phantom.

The study was conducted using a human aorta phantom modelled on a patient with an abdominal aortic aneurysm. To perform the scans of the aorta phantom, it was placed in a specially designed, sealable perspex container, which was filled with iodinated contrast medium. A series of scans was performed using different protocols to test the effect on dose and image quality. These were: the variable detectors (4-, 32- and 64-slice) used in image acquisition, x-ray tube current $(\mathrm{mA})$ and pitch values.

A line profile analysis was conducted at three separate locations for each different protocol, namely: the renal artery, superior mesenteric artery, and abdominal aortic aneurysm to determine the degree of image artefacts using standard deviation, which was measured on the virtual intravascular endoscopy images. The effective dose was also calculated for each scanning protocol.

The results indicate that the scans performed on 64 detectors multi-detector CT produce better image quality compared to 32 and 4 detectors. Increasing scanning parameters such as $\mathrm{mA}$ enhances image quality but it also increases the patient's radiation dose. It is also noted that the pitch is inversely proportional to radiation dose, resulting in degradation of image quality. From these results, a 64 multi-detector CT scanner with a scanning protocol of $120 \mathrm{kVp}, 70 \mathrm{~mA}$, and a pitch of 0.641 is ideal for imaging of abdominal aortic aneurysms because it allows for fewer artefacts and less radiation dose when compared to other scanning protocols.
\end{abstract}

Keywords: aneurysm, artefacts, image quality, multi-detector scan, radiation dose

\section{Introduction}

The use of endovascular stent grafts has redefined the traditional method of treatment for abdominal aortic aneurysm (AAA). This technique has been widely used in many medical centres as an effective alternative to conventional open surgery, especially in patients with co-morbid medical conditions. ${ }^{1,2}$ Unlike open surgical repair, the success of endovascular repair relies mainly on medical imaging. Helical computed tomography angiography (CTA) has been reported as minimally invasive techniques in both the preoperative planning and postoperative follow-up..$^{3-7}$ the recent emergence of multi-detector computed tomography (MDCT) has demonstrated numerous potential advantages compared with conventional single slice $\mathrm{CT}$ and invasive angiography. These include minimal invasiveness, high accuracy for detection of stenoses and dilative disease and complete visualisation of the entire arterial branches.$^{8-10}$ However, the major drawbacks of MDCT are patient radiation exposure due to suboptimal scanning protocols. For this reason, the optimisation of radiation dose during MDCT scanning has been the topic of many recent studies involving chest disease, pediatric assessment and CT colonography. ${ }^{11-13}$

The purpose of this study was to investigate the influence of radiation dose on image quality of MDCT angiography in preaortic stent grafting, visualised by $3 \mathrm{D}$ CT virtual intravascular endoscopy (VIE) based on a human aorta phantom.

\section{Materials and methods}

\section{Human aorta phantom}

The study was conducted using a human aorta phantom. ${ }^{14}$ The phantom was built using medical rapid prototyping and was based on a typical patient with an AAA. All surrounding structures were excluded from the phantom, with the exception of the superior mesenteric artery (SMA), the renal arteries and the aortic aneurysm.

To perform the scans of the aorta phantom, it was placed in a specially designed, sealable perspex container. The container was filled with iodinated contrast medium (Omnipaque 350), which was diluted to $4 \%$ in order to produce 370 Hounsfield Units (HU), similar to that used in routine abdominal CT scanning.

\section{CT scanner parameters}

The scanner used for this study was a Toshiba Aquilion, 64 detector CT scanner (Toshiba Medical Systems, The Netherlands). The detector collimation of the scanner is $64 \times 0.5 \mathrm{~mm}$. The rotation time of the scanner was $0.5 \mathrm{sec}$ and the tube voltage was 120 $\mathrm{kVp}$. The automatic dose modulator was turned off in order to keep the mA consistent for each scan. The field of view was kept constant at $180 \mathrm{~mm}$, and a constant scan length of $110 \mathrm{~mm}$ was used with a matrix of $512 \times 512$. 
Optimisation of radiation dose and image quality in $3 D$ multi-detector CT angiography in abdominal aortic aneurysm: an in vitro aorta phantom study

Table 1 Scanning protocols of MDCT angiography in the aorta phantom study

Test cone beam artefact by variable detectors

\begin{tabular}{|c|c|c|c|c|}
\hline Slice thickness $x$ No. of detectors & Pitch & $\mathbf{k V p}$ & $\mathrm{mA}$ & ED (mSv) \\
\hline $64 \times 0.5$ & 0.75 & 120 & 47 & 2.34 \\
\hline $32 \times 0.5$ & 0.75 & 120 & 47 & 2.19 \\
\hline $4 \times 0.5$ & 0.75 & 120 & 47 & 3.88 \\
\hline
\end{tabular}

Test effects of dose on image quality

Slice thickness $x$ No. of detectors

$64 \times 0.5$

Pitch

0.75

0.75

0.75

0.75

$64 \times 0.5$

$64 \times 0.5$

Test effects of pitch on image quality

Slice thickness $x$ No. of detectors

$64 \times 0.5$

$64 \times 0.5$

$64 \times 0.5$

$64 \times 0.5$
Pitch

0.641 Detail

0.75 Original

0.828 Standard

1.484 Fast

$\mathbf{k V p}$
120
120
120
120

kVp

$\mathbf{m A}$

30

50

70

100
ED (mSv)

1.00

1.67

2.34

3.34

$\mathrm{ED}=$ effective dose

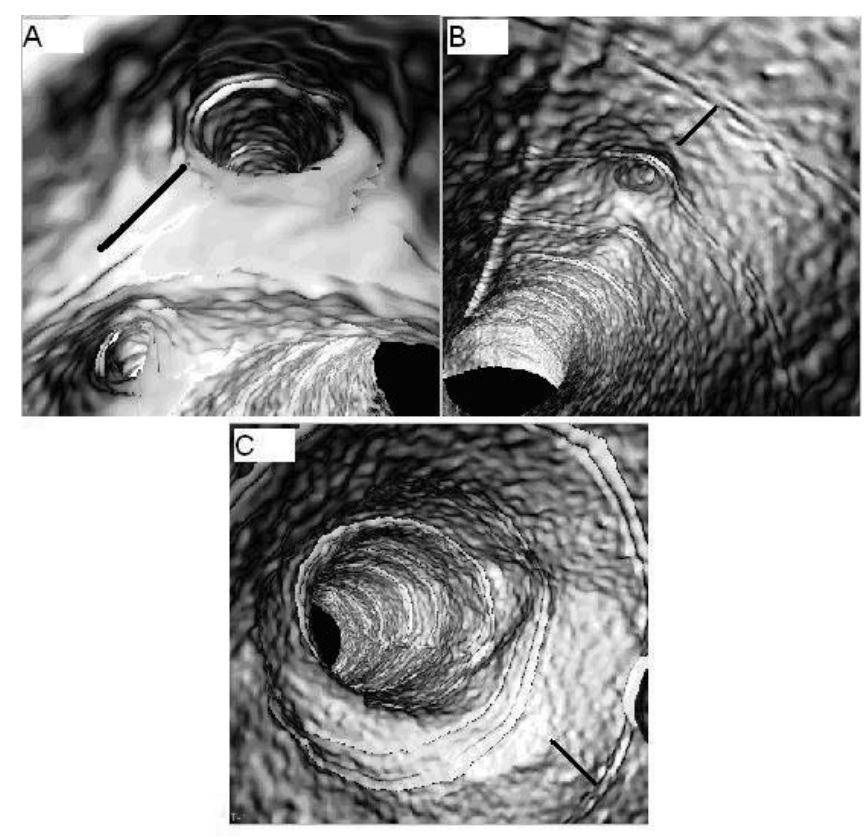

Figure 1 VIE images were measured in three anatomic locations, including SMA (A), right renal artery (B) and aortic aneurysm (C). Lines indicate the region of interest for measuring the pixel intensity.

For scanning, the longitudinal axis of the phantom was placed parallel to the longitudinal axis of the gantry. Image data were acquired with different specifications according to the different scan protocols.

Table 1 shows the scan protocols used in this study. Note that each scan sequence has only one variable whilst all other factors were kept constant for ease of comparison in determining the optimal scan protocol for CTA of AAA. The parameters used were dependent on the Toshiba scanner used to carry out the study.

After scanning, the CT data was burned onto computer disks and transferred to a workstation equipped with Analyze V 6.0

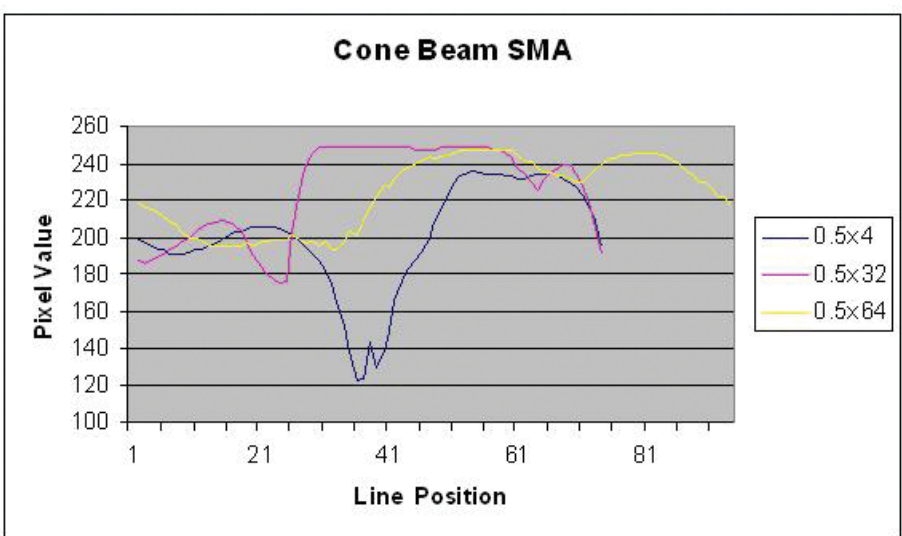

Figure $2 \mathrm{~A}$ line profile shows the pixel intensity values measured at the SMA acquired with variable scanning protocols.

(www.analyzedirect.com, Mayo Clinic, USA) for data processing. A total of nine volume data sets were generated.

\section{Image analysis and assessment}

From each of the volume data sets, VIE images of the SMA, renal arteries and the AAA were generated (Figure 1). An upper threshold of $307 \mathrm{HU}$ was selected for the removal of the contrast medium from the aorta, allowing visualisation of the internal surfaces of the aortic ostium and arterial wall.

The quantification of artefacts was based on the pixel intensity measured on each surface rendered VIE image. For greater accuracy, consistent settings were required to keep the VIE images identical in demonstrating each anatomical feature in all scanning protocols. This requirement was achieved by saving the camera's spatial location (X, Y, Z coordinates) for each of the three VIE views from one data set $(64 \times .05)$, allowing the remaining data sets to demonstrate the same views by loading the previously saved camera positions.

Ideally, the internal surface of the aorta should appear smooth 
Table 2 SD measured on the VIE images generated from variable protocols

ROI

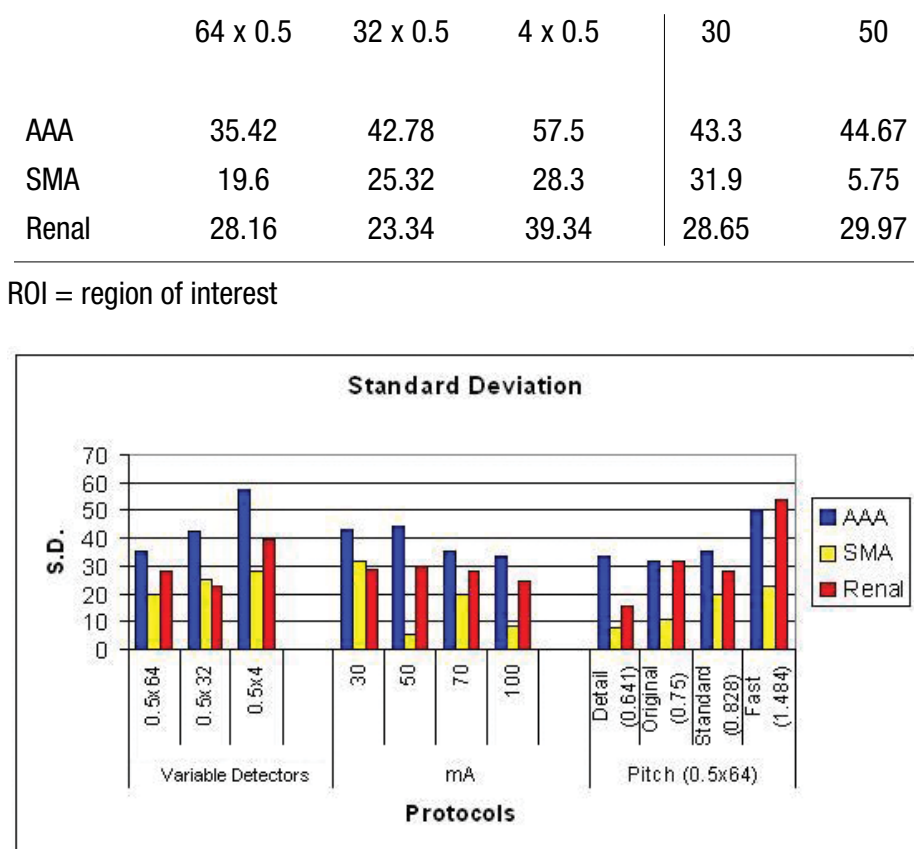

Figure 3 SD measured with variable detector protocols, $\mathrm{mA}$ and pitch values.

in a VIE image, free from artefacts. To measure the degree of stair-step artefacts present, an objective numerical approach was taken. For each VIE image, a line profile analysis was performed which provided the pixel intensity values along a prescribed path. Consequently, the standard deviation (SD) of the pixel value was measured to determine the degree of artefacts. A higher SD indicated a greater variation of pixel intensities, which is consistent with the greater presence of artefacts.

Consistent line profiles were drawn across each of the images and the pixel intensity values were also recorded. These pixel intensity values were then transferred into Microsoft Excel ${ }^{\circledR}$ to produce a plot of pixel intensities versus pixel locations along a prescribed line. This graphic representation of pixel values also enabled us to assess the degree of artefacts present (Figure 2).

To calculate the effective dose vs. scanning protocols, the dose length product (DLP) values $(\mathrm{mGycm})$ was converted into effective dose values $(\mathrm{mSv})$ using the following formula: ${ }^{15}$

$\mathrm{ED}=\mathrm{E}_{\mathrm{DLP}} \times \mathrm{DLP}$

whereby $E D$ : Effective Dose,

$\mathrm{E}_{\mathrm{DLP}}$ : Normalised Effective Dose (0.015 for abdomen),

$D L P$ : Dose Length Product

The calculated effective dose values are a broad estimate and will not be entirely accurate.

\section{Statistical analysis}

The results were analysed using SPSS V 14.0 (SPSS Inc, Chicago, Il) and a linear regression analysis was performed to investigate the relationship between the SD, radiation dose and variable scanning parameters, in an effort to determine the optimal scanning parameter for CTA of AAA.

\section{Results}

Table 2 shows the SD. measured from the VIE images generated from different scanning protocols, while Figure 3 presented these measurements in bar charts. The SD. measured in the variable detector protocol shows a general pattern where the SD.
mA

Pitch (64 x 0.5)

\begin{tabular}{cc|cccc}
70 & 100 & $\begin{array}{c}\text { Detail } \\
(0.641)\end{array}$ & $\begin{array}{c}\text { Original } \\
(0.75)\end{array}$ & $\begin{array}{c}\text { Standard } \\
(0.828)\end{array}$ & $\begin{array}{c}\text { Fast } \\
(1.484)\end{array}$ \\
35.42 & 34.04 & 33.79 & 32.4 & 35.42 & 50.41 \\
19.6 & 8.6 & 7.99 & 11.44 & 19.6 & 23.29 \\
28.16 & 24.56 & 15.71 & 32.3 & 28.16 & 54.34
\end{tabular}

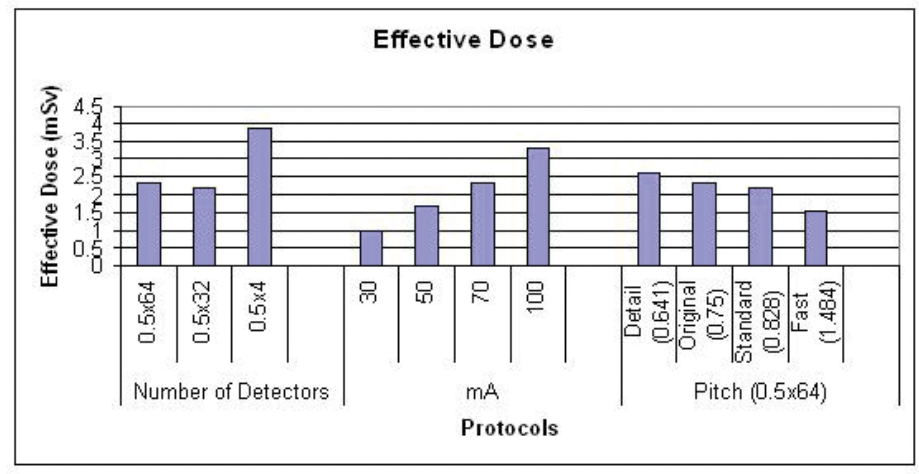

Figure 4 Effective radiation dose measured with variable detector protocols, $\mathrm{mA}$ and pitch values.

values of the $64 \times 0.5$ and $32 \times 0.5$ scans were less than those measured in the $4 \times 0.5$ scans. The measurements in the AAA and the SMA demonstrated the lowest SD with the protocol of 64 $\mathrm{x} .05$ as the protocol being 35.4 and 19.6 respectively. The $32 \mathrm{x}$ 0.5 protocol provided the best image quality for the visualisation of renal artery, with the lowest SD value of 23.3. In contrast, the $64 \times 0.5$ protocol of VIE at the renal artery resulted in S.D. value of 28.2 .

For the different $\mathrm{mA}$ protocols, the same VIE mages were created and measurements were taken. There was a common trend where the SD values measured in the level of renal artery and AAA with the $30 \mathrm{~mA}$ and $50 \mathrm{~mA}$ were higher than those measured in the $70 \mathrm{~mA}$ and $100 \mathrm{~mA}$ scans. The averaged $30 \mathrm{~mA}$ and $50 \mathrm{~mA}$ SD values were 35.9 and 37.3 respectively; whilst the SD. in the $70 \mathrm{~mA}$ and $100 \mathrm{~mA}$ were significantly lower with 31.8 and 29.3. In the location of SMA, the SD generally decreased with the increase of $\mathrm{mA}$, except with $50 \mathrm{~mA}$, which showed a significantly lower than that measured with $70 \mathrm{~mA}$ and $10 \mathrm{~mA}$.

Various pitch protocols were used in the study to determine which produces the lowest degree of artefacts (lowest SD). As shown in Table 2, the SD measured with the fast pitch always produced the highest SD when compared to that measured with other three pitch values.

Figure 4 demonstrates that the effective doses measured with the $64 \times 0.5$ and $32 \times 0.5$ detector scans are fairly similar and have $\mathrm{mSv}$ values of 2.34 and 2.19 respectively. This difference of 0.15 $\mathrm{mSv}$ shows the similarity of the two scans in terms of radiation exposure. The $4 \times 0.5$ detector scan had a dose of $3.88 \mathrm{mSv}$ and it is significantly higher than that measured with the other two scans.

The results showed that the effective dose increased with the increase of $\mathrm{mA}$ values, as shown in Table 1, which ranged from $1.0 \mathrm{mSv}$ to $3.34 \mathrm{mSv}$. However, image quality was improved with higher $\mathrm{mA}$ values. Figure 5 shows the VIE images acquired with $30 \mathrm{~mA}$ and $100 \mathrm{~mA}$, and the artefacts were less apparent when 


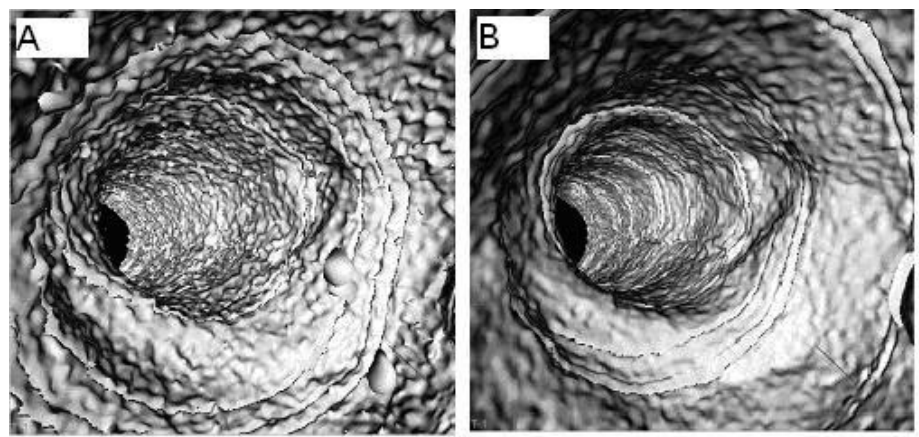

Figure 5 VIE images of viewing the aortic aneurysm wall were acquired with a scanning protocol of $64 \times 0.5$, pitch 0.828 , and $\mathrm{mA} 30(\mathrm{~A})$ and $100(\mathrm{~B})$. It is clearly shown that image quality is significantly improved when image was generated with $100 \mathrm{~mA}$ with fewer artifacts. Lines indicate the region of interest for measuring the pixel intensity.
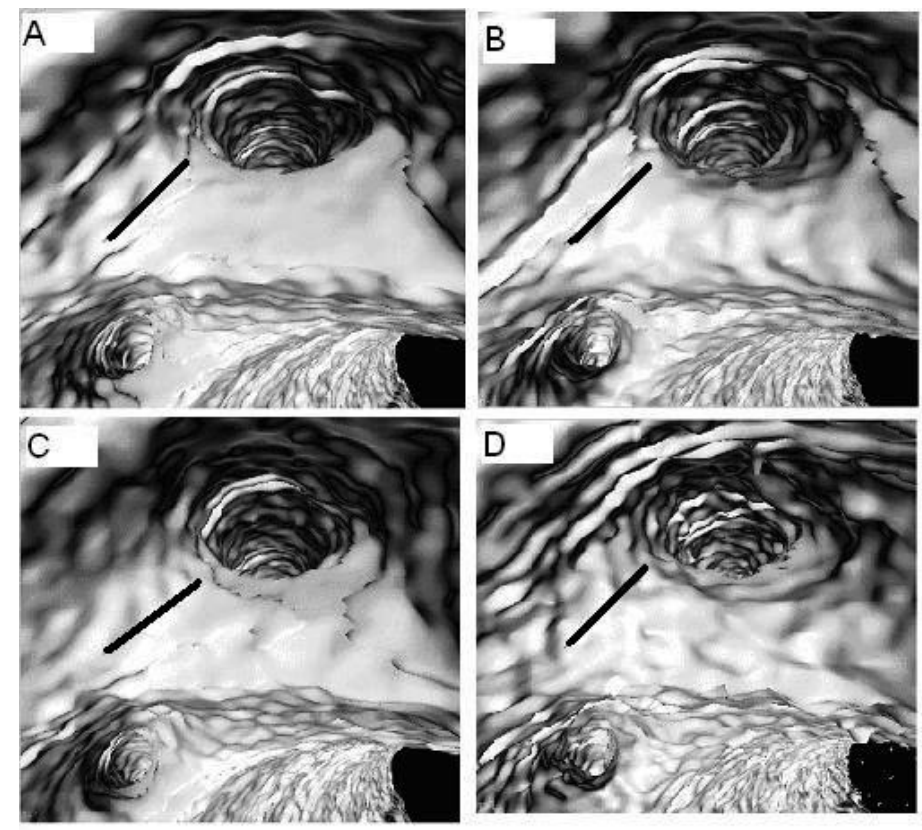

Figure 6 VIE images of the SMA acquired with scanning protocol of $64 \times 0.5$ and pitch values of (A) 0.641 , (B) 0.828 , (C) 0.75 and (D) 1.484 respectively. The SD increased from 7.99 to 23.29 when pitch increased from 0.641 to the fast level D.

compared the VIE image generated with $100 \mathrm{~mA}$ to that with $30 \mathrm{~mA}$.

Changing the pitch value in the protocols affected the resultant effective dose. As observed in the current study, the fast scan had the lowest radiation dose and the detail scan the highest dose. Figure 6 shows a series of VIE images generated with variable pitch values. The artefacts became apparent when the pitch increased to 1.484 (Figure 6D).

Figures 7 and 8 demonstrated the linear relationship between SD measured in renal artery vs and radiation dose vs pitch values, which is significant difference.

\section{Discussion}

CTA is non-invasive, easy to perform, less costly, and accompanied by minimal complications comparing with digital subtraction angiography (DSA). Several studies have investigated the diagnostic performance of MDCT angiography for the assessment of abdominal aorta. MDCT is superior to DSA in the preoperative assessment of AAA. ${ }^{16}$

Studies performed by researchers showed that CTA has high sensitivity and specificity for detecting aneurysms. ${ }^{16-18}$ They

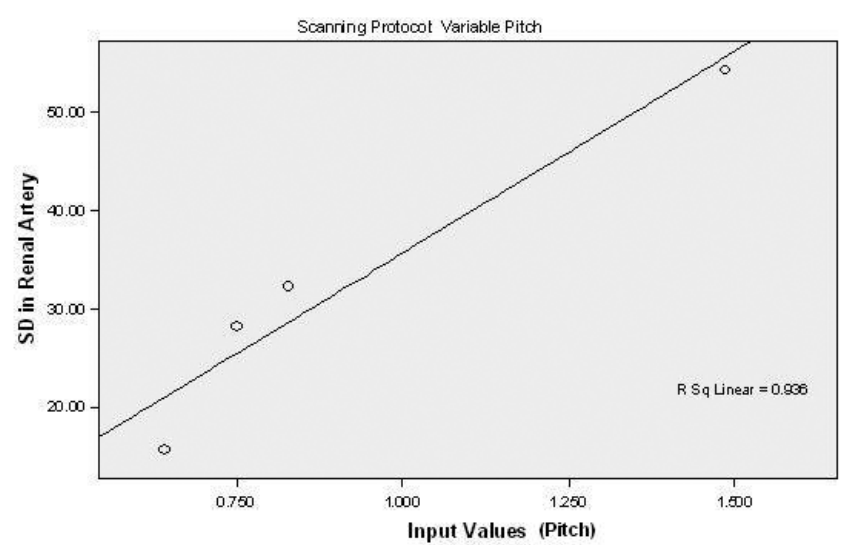

Figure 7 Scatterplot shows significant direct relationship between the SD measured in renal artery vs pitch value.

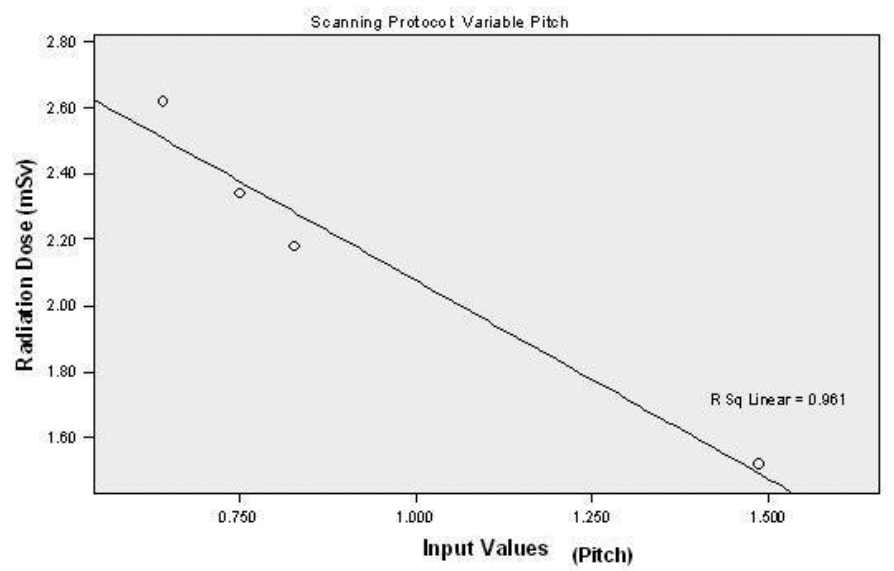

Figure 8 Scatterplot shows significant inverse relationship between radiation dose and pitch values.

claimed that CTA has the ability to depict bony landmarks and proximity of the aneurysm to other vascular structures. This is important in treatment planning. ${ }^{18}$ Recent development of 64-slice $\mathrm{CT}$ in the imaging of aneurysms has further improved the imaging quality, by increasing both spatial and temporal resolution. ${ }^{19}$

In the study, three common factors (variable detectors, $\mathrm{mA}$ and pitch) that affect radiation dose with regards to image quality were evaluated.

The results indicate that 64 detectors produce better image quality compared to the 32 and 4 detectors. This is consistent with the expectation that the more detectors used in image acquisition, the better the image quality. Fishman ${ }^{20}$ concluded in his study that using a 64 slice MDCT provides optimal spatial resolution, enables more detailed vascular mapping, more accurate stenosis measurement, better soft tissue detail and ultimately optimisation of data available for $3 \mathrm{D}$ visualisation.

There is a significant increase in effective dose when 4 detectors MDCT is used, compared with 32 and 64 detectors MDCT. This contradicts with the expectations that 64 detectors MDCT may have higher effective radiation dose than 4 detectors MDCT. A possible explanation for this contradiction is the significantly longer scanning time (42 seconds) taken in the study for 4 detectors compared to the 32 and 64 detectors (10 seconds and 7 seconds respectively) with the same section thickness $(0.5 \mathrm{~mm})$. This is usually not the case in clinical environment, as a thicker slice thickness will be used for a 4 detector scan in order to cover the same scanning length as that obtained with 32 and 64 detectors, thus the scanning time will be similar. Therefore, the results 
should be interpreted with caution.

Increasing technical factors such $\mathrm{mA}$ enhances image quality but as a consequence it increases patients' radiation dose. ${ }^{21}$ Any increase in radiation dose must be justified, otherwise it should be minimised to ensure that the patient is not subjected to unnecessary exposure. In many cases, further increase in radiation dose does not necessarily improve image quality or the diagnostic quality of the examination. ${ }^{22}$

In the present study, the effective dose has increased more than three-fold when the $\mathrm{mA}$ is increased from 30 to 100 (Figure 4 ), however, the SD value decreases by 6.68 , which is minimal. This indicates that decreasing the $\mathrm{mA}$ during scanning will not compromise the image quality required for clinical diagnosis. The mild increase in diagnostic quality does not justify the three-fold increase in radiation dose.

However, subjective assessment of the images shows a significant difference in image quality that could make a difference in diagnosis (Figure 6). It is uncertain how much change in SD value will cause a significant difference in subjective image quality as this assessment was not performed in the present study. Further studies on subjective assessments of the images are strongly recommended.

It is well known that as the pitch increases, radiation dose decreases, however, at the same time image quality will be compromised (Figure 6). The results further support this, whereby there is an inverse relationship between the degree of artefacts and effective dose when pitch is altered (Figures 7,8).

There were a number of limitations in the study which should be addressed. First, it must be appreciated that the phantom was not designed to exactly replicate real aortic tissue. The phantom wall was constructed out of a dense material (perspex) which resulted in a higher CT number of approximately $170 \mathrm{HU}$, compared with approximately $40 \mathrm{HU}$ for aortic tissue. The internal surfaces of the phantom did not simulate the smooth muscle of an abdominal aorta due to tiny impurities. This complicated results as the 64 detectors data were providing higher SD readings, which were not indicating a greater presence of artefacts, but actually the detection of greater detail.

Second, the study results were obtained from a static contrast fluid model. In the real environment where contrast enhanced blood is flowing, the contrast density varies along the vessel, which can affect image quality. No subjective image analysis was performed in this study, which is another limitation. Initially, data was acquired to test the effects of raw slice thickness on image quality, however, these results were omitted from the study due to a lack of data and time.

Finally, measurement of the SD is operator-dependent and thus highly subjective. Assessment of inter-or intra-observer agreement needs to be considered when performing these measurements.

\section{Conclusion}

In conclusion, a human aortic phantom was successfully used to investigate the relationship of radiation dose and image quality in 3D multi-detectors CTA. Based on the results, a 64 MDCT scanner with a scanning protocol of $120 \mathrm{kVp}, 70 \mathrm{~mA}$, and a pitch of 0.641 is ideal for imaging of the abdominal aortic aneurysm enabling the generation of fewer artefacts and less radiation dose when compared to other scanning protocols in the study. However, further studies should be performed in order to further support the results before recommendations for clinical practice can be made.

\section{Acknowledgement}

The authors would like to thank Mr Grant Melloncelli, (CT technologist, St John of God Hospital, Subiaco) for his help in scanning the phantom. Great appreciation is given to Mr Gil Stevenson for his assistance in the statistical analysis of the results.

\section{References}

1 Chuter, TA, Wendt G, Hopkinson BR et al. Bifurcated stent-graft for abdominal aortic aneurysm. Cardiovasc Surg 1997; 5: 388-92.

2 Parodi JC, Palmaz JC, Barone HD. Transfemoral intraluminal graft implantation for abdominal aortic aneurysm. Ann Vasc Surg 1991; 5: 491-99.

3 Moritz JD, Rotermund S, Keating DP, Oestmann JW. Infrarenal abdominal aortic aneurysms: implications of CT evaluation of size and configuration for placement of endovascular aortic grafts. Radiology 1996; 198: 463-46.

4 Ecklund K, Hartnell GG, Hughes LA, Stockes K, Finn JP. MR angiography as the sole method in evaluating abdominal aortic aneurysms: correlation with conventional techniques and surgery. Radiology 1994; 192: 345-50.

5 Siegel CL, Cohan RH. CT of abdominal aortic aneurysms. Am J Roentgenol 1994; 163: 17-29.

6 Kaufman JA, Geller SC, Petersen MJ, Cambria RP, Prince MR, Waltman AC. MR imaging (including MR angiography) of abdominal aortic aneurysms: comparison with conventional angiography. Am J Roentgenol 1994; 163: 203-10.

7 Rubin GD, Dake MD, Napel SA, McDonnell CH, Jeffrey RB Jr. Three-dimensional spiral CT angiography of the abdomen: initial clinical experience. Radiology 1993; 186: 147-52.

8 Catalano C, Fraioli F, Langhi a et al. Infrarenal aortic and lower extremity disease: diagnostic performance of multidetector row CT angiography. Radiology 2004; 231: 555-61.

9 Ofer A, Niteky S, Linn S et al. Multidetector CT of peripheral vasculature disease: a prospective comparison intraarterial digital subtraction angiography. Am J Roentgenol 2003; 180: 719-24.

10 Martin ML, Tay KH, Flak B et al. Multidetector CT angiograph of the artoiliac system and lower extremities: a prospective comparison with digital subtraction angiography. Am J Roentgenol 2003; 180: 1085-91.

11 Verdun FR, Lepori D, Monnin P et al. Management of patient dose and image noise in routine pediatric CT abdominal examinations. Eur Radiol 2004; 14: $835-41$

12 Iannaccone R, Langhi A, Catalano C et al. Detection of colorectal lesions: lower-dose multidectector row helical CT colonography compared with conventional colonoscopy. Radiology 2003; 229: 775-81.

13 Diderich S, Thomas M, Semik et al. Screening for early lung cancer with lowdose spiral computed tomography: results of annual follow-up examination in asymptomatic smokers. Eur Radiol 2003; 14: 691-02.

14 Sun Z, Winder J, Kelly B, Ellis P, Kennedy P, Hirst D. Influence of scanning parameters of single slice helical CT angiography on image quality of VIE: in vitro phantom study. Comput Med Imaging Graph 2004, 28 (3): 1215

15 Rothenberg LN 2006, Doses and Dose Calculations, Health Physics Society, viewed 1 October 2006, <http://www.hps.org/publicinformation/ate/q5194. htm>.

16 Rydberg J, Kopecky KK, Lalka SG et al. Stent grafting of abdominal aortic aneurysms: pre-and postoperative evaluation with multislice helical CT. $J$ Comput Assist Tomogr 2001; 25: 580-86.

17 Jacob, J. How to do coronary CT angiography: a radiologist's perspective. Supplement to Appl Radiol 2005; 34-43.

18 Jayaraman $\mathrm{M}$ et al. Detection of intracranial aneurysms: multi-detector row angiography compared with DSA Radiology 2004; 230: 510-518.

19 Flohr T, Stierstorfer K, Raupach R, Ulzheimer S, Bruder H. Performance evaluation of a 64-slice CT system with z-flying focal spot. Rofo 2004; 176: 1804-10.

20 Fishman E. Introduction to 64-detectors CT and its role in coronary imaging, Supplement to Appl Radiol, December 2005.

21 Reddinger W. CT image quality. OutSource Inc; 1998. pp 1-44.

22 Fraioli F et al. Low dose multidetector - row CT angiography of the infra - renal aorta and lower extremity vessels: image quality and diagnostic accuracy in comparison with standard DSA Eur Radiol 2006; 16: 137-46. 